\title{
Childhood Fibrillary Astrocytoma
}

National Cancer Institute

\section{Source}

National Cancer Institute. Childhood Fibrillary Astrocytoma. NCI Thesaurus. Code C114963.

A fibrillary astrocytoma that occurs during childhood. 